\title{
FAST ROOM TEMPERATURE DETECTION OF STATE OF CIRCULAR POLARIZATION OF TERAHERTZ RADIATION
}

\author{
S. D. Ganichev, H. Ketterl, and W. Prettl \\ Institut für Experimentelle und Angewandte Physik \\ Universität Regensburg \\ D 93040 Regensburg, Germany
}

Received March 19, 2003

\begin{abstract}
We report on a room temperature detector which allows to determine and monitor the state of polarization of terahertz radiation with picosecond temporal resolution. The detector is based on the circular photogalvanic effect recently observed in GaAs/AlGaAs quantum wells. The circular photogalvanic effect yields in response to elliptically polarized radiation a current signal proportional to the degree of circular polarization. The peak current signal occurs in unbiased samples for circular polarization, vanishes at linear polarization and changes sign by switching the helicity from right-handed to left-handed. The detector consists of a (113)A MBE grown pGaAs/AlGaAs multiple quantum well structure. The response has been measured in the wavelength range between $76 \mu \mathrm{m}$ and $280 \mu \mathrm{m}$ at normal incidence of the radiation on the sample.
\end{abstract}

Key words: circular photogalvanic effect, fast infrared detection, helicity of radiation

\section{Introduction}

Room temperature detection of short $\mathrm{THz}$ laser pulses is possible using pyroelectricity [1], bulk semiconductor devices like photon drag detectors [2-5], Schottky-diodes [5], and intraband photoconductivity [6]. Recently low dimensional semiconductor structures and superlattices have been demonstrated to be very efficient for detection $\mathrm{THz}$ radiation with high temporal resolution. In particular photovoltaic [7], photon-drag [8], and hot electrons [9] effects in quantum well $(\mathrm{QW})$ structures as well as $\mathrm{THz}$ radiation driving superlattices [10] are promising means of large bandwidth detection. Here

847 
we report on a new approach to $\mathrm{THz}$ detection applying the circular photogalvanic effect which has recently been observed in GaAs based quantum wells $(\mathrm{QW})[11,12]$. Circularly polarized radiation generates a current in the unbiased sample. Because the photogalvanic effect does not involve any charge separation, space charge regions or gradient-induced drift currents, photogalvanic detectors have the advantages of fast transient signal response at low impedance. The only physical speed limitations result from momentum relaxation times, which are in order of picoseconds at room temperature. The striking feature of this photogalvanic effect is that the current flows perpendicular to the radiation propagation and that the sign of the current reverses by changing the helicity of the radiation from right-handed to left-handed. Therefore the circular photogalvanic effect does not only trace the time dependence of a radiations pulse but it also gives the state of polarization of the electromagnetic field in a very direct way.

\section{Physical Background}

The photogalvanic effects arise in homogeneous samples of noncentrosymmetric media [13] under homogeneous excitation due to an asymmetry of the interaction of free carriers with photons, phonons, static defects or other carriers. In the THz-range the circular photogalvanic effect occurs at intersubband or intrasubband transitions in quantum-well structures. Phenomenologically, the effect is a transfer of the angular momentum of circularly polarized photons into a directed motion of free carriers, electrons or holes. Therefore the effect depends on the symmetry of the medium.

The photogalvanic current under study can be described by the following phenomenological expression [11]:

$$
j_{\lambda}=\sum_{\mu \nu} \chi_{\lambda \mu \nu}\left(E_{\mu} E_{\nu}^{*}+E_{\nu} E_{\mu}^{*}\right) / 2+\sum_{\mu} \gamma_{\lambda \mu} i\left(\boldsymbol{E} \times \boldsymbol{E}^{*}\right)_{\mu},
$$

where $\boldsymbol{E}$ is the complex amplitude of the electric field of the electromagnetic wave and $i\left(\boldsymbol{E} \times \boldsymbol{E}^{*}\right)=\hat{\mathrm{e}} P_{\text {circ }} E_{0}^{2}$ with the degree of circular polarization $P_{\text {circ }}=\left(\left|E_{+}\right|^{2}-\left|E_{-}\right|^{2}\right) /\left(\left|E_{+}\right|^{2}+\left|E_{-}\right|^{2}\right)$ where $E_{+}$and $E_{-}$are the amplitudes of left and right handed circularly polarized radiation, respectively. $E_{0}=|E|$ is the electric field amplitude and ê is the unit vector pointing in the direction of the light propagation. 
The photocurrent given by the tensor $\chi$ describes the so-called linear photogalvanic effect (LPGE) because it is usually observed under linearly polarized optical excitation. The circular photogalvanic effect (CPGE) yielding the helicity dependent current is described by the pseudo-tensor $\gamma$ and can be observed only under circularly polarized excitation.

A symmetry analysis shows that in order to obtain a helicity dependent photoresponse at normal incidence, which is convenient for detection, the symmetry of the structure must be reduced to the point group $\mathrm{C}_{s}$. This group contains only two elements: the identity transformation and one mirror reflection. This can easily be obtained in zinc-blende based QW structures by choosing a suitable crystallographic orientation. This condition is met, for instance, in (113) grown structures. In this case the helicity dependent current flows in the direction $x \|[1 \overline{1} 0]$ being normal to the mirror plane which contains the [110] axis. For the light initially polarized along $x$ and transmitted through the $\lambda / 4$ plate, as in experiments described below, we have

$$
j_{x}=\gamma_{x z} t_{p} t_{s} \cos \Theta E_{0}^{2} P_{c i r c}
$$

where $t_{p}$ and $t_{s}$ are transmission coefficients after Fresnel's formula for linear $p$ - and $s$-polarizations, $\Theta$ is the angle of refraction defined by $\sin \Theta=\sin \Theta_{0} / n, \Theta_{0}$ is the angle of incidence and $n$ is the index of refraction.

Microscopically, the CPGE in MQWs is due to optical spin orientation of charge carriers and asymmetric spin-dependent scattering of spin-polarized carriers followed by an appearance of an electric current [12]. The two states of light circular polarization result in different spin orientations and, thus, in electric photocurrents of opposite directions. The principal aspect of a photon helicity driven current is the removal of spin-degeneracy in the subband states due to the reduced symmetry of the quantum well structure $[12,14]$. It is related to the appearance of $\mathbf{k}$-linear terms in the Hamiltonian

$$
H^{(1)}=\sum_{l m} \beta_{l m} \sigma_{l} k_{m}
$$

where the real coefficients $\beta_{l m}$ form a pseudo-tensor subjected to the same symmetry restrictions as the pseudo-tensor $\gamma ; \sigma_{l}$ are Pauli spin matrices. Spin degeneracy results from the simultaneous presence of 
time-reversal and spatial inversion symmetry. If one of these symmetries is broken the spin degeneracy is lifted. In (113)-grown GaAs QW systems considered here the spatial inversion symmetry is broken (the point group $\mathrm{C}_{s}$ does not contain the inversion operation) and, as a consequence, spin-dependent and $\mathbf{k}$-linear terms appearing in the electron Hamiltonian lead to a splitting of the electronic subbands at a finite in-plane wave vector. As discussed in [14] the coupling between the spin of the carriers $\left(\sigma_{l}\right)$ and the carrier momentum $\left(k_{m}\right)$ together with spin-controlled dipole selection rules yields a net current under circularly polarized excitation. Depending on the band structure of quantum wells and the photon energy this spin photocurrent can be either due to direct or indirect intersubband transitions.

\section{Experimental}

The concept of the CPGE-detector was realized using quantum well structures grown by molecular beam epitaxy on semi-insulating (113)oriented GaAs substrates. The samples were $p$-GaAs/AlGaAs multiple QWs containing 20 wells of $15 \mathrm{~nm}$ width. Samples with free hole densities of $2 \cdot 10^{11} \mathrm{~cm}^{-2}$ were studied at room temperature. A pair of

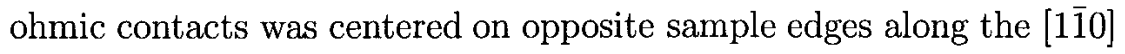
axis (see inset in Fig. 1).

A high power pulsed $\mathrm{THz} \mathrm{NH}_{3}$-laser [15] has been used as radiation source delivering $100 \mathrm{~ns}$ pulses with radiation power $P$ up to $100 \mathrm{~kW}$. Several lines of the $\mathrm{NH}_{3}$-laser between $\lambda=76 \mu \mathrm{m}$ and $280 \mu \mathrm{m}$ have been applied for excitation in the $\mathrm{THz}$ range. Depending on the frequency the $\mathrm{THz}$ radiation induces direct optical transitions between heavy hole and light hole subbands and indirect optical transitions in the lowest heavy hole subband. The laser light polarization was modified from linear to circular using quartz $\lambda / 4$ plates. The helicity of the incident light was varied according to $P_{\text {circ }}=\sin 2 \varphi$ where $\varphi$ is the angle between the initial plane of linear polarization and the optical axis of the $\lambda / 4$ plate.

With irradiation of (113)-oriented samples of $\mathrm{C}_{s}$ symmetry a current signal proportional to the helicity $P_{\text {circ }}$ is observed under normal incidence. We note that the samples were unbiased, thus the irradiated samples represent a current source. A voltage signal is obtained by applying a $50 \Omega$ load resistor in a closed circuit. The response follows the temporal structure of the laser pulse intensity and changes 


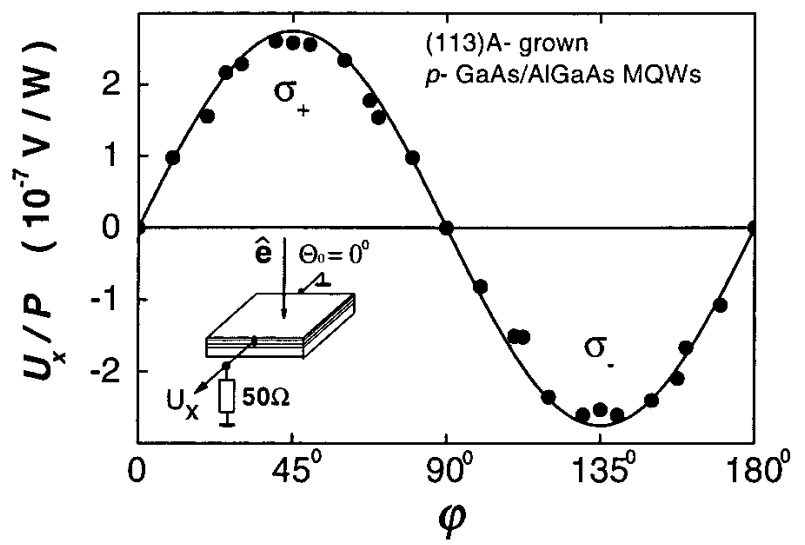

Fig. 1. Photoresponse of $p$-type (113)A-grown GaAs/AlGaAs QWs structure normalized by the light power $P$ as a function of the phase angle $\varphi$ defining helicity. Measurements are presented for $T=300 \mathrm{~K}$ and normal incidence of radiation at $\lambda=76 \mu \mathrm{m}$. The current $j_{x} \propto U_{x}$ flows along [110]- direction perpendicular to the mirror plane of the $C_{s}$ symmetry. Full lines are fitted to Eq. (1) using one $y$-axis scaling parameter. The inset shows the geometry of the experiment.

sign if the circular polarization is switched from left-handed to righthanded (Fig. 1). The direction of the current is determined by the symmetry of the crystal. The helicity dependent current flows always along the $[1 \overline{1} 0]$ - direction perpendicular to the plane of mirror reflection of the point group $\mathrm{C}_{s}$, independent of the plane of incidence of the laser beam. In Fig. 2 we take a closer look on the dependence of the photoresponse on the angle of incidence $\Theta_{0}$ of the circularly polarized laser beam. Comparison of Eqs. (1) and (2) and Figs. 1, 2 shows a good agreement between the theory and the experimental data.

In the wavelength range from $76 \mu \mathrm{m}$ to $280 \mu \mathrm{m}$ the voltage response of the CPGE detector depends linearly on the power up to highest intensities applied $\left(2 \mathrm{MW} / \mathrm{cm}^{2}\right)$. The sensitivity in the range of $0.2 \mu \mathrm{V} / \mathrm{W}$ at $50 \Omega$ is low but enough to detect short $\mathrm{THz}$ pulses of sources like optically pumped molecular lasers and free-electron-lasers. It can be improved by using a larger number of quantum wells. Note that even in a large number of QWs the extinction of radiation is very 


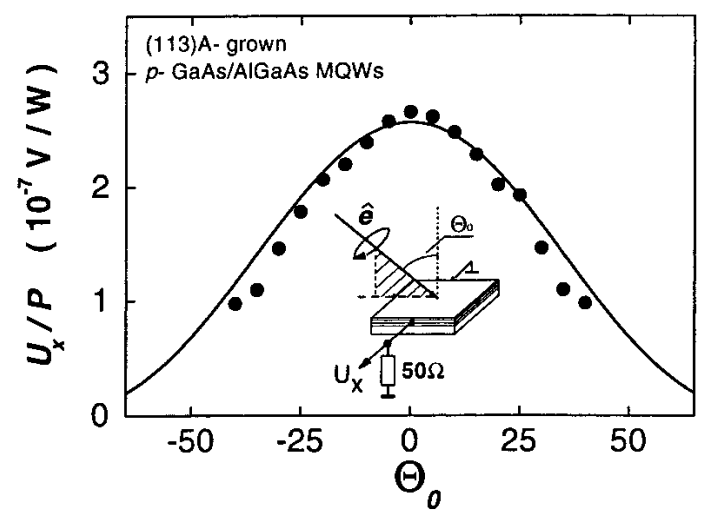

Fig. 2. Photoresponse of 20 -type (113)A- grown GaAs/AlGaAs QWs normalized by the light power $P$ as a function of the angle of incidence $\Theta_{0}$ for right circularly polarized radiation measured perpendicular to light propagation $(T=300 \mathrm{~K}, \lambda=76 \mu \mathrm{m})$. Full line is fitted to Eq. (2) using one $y$-axis scaling parameter.

low, hence the device can be used to monitor the state of circular polarization of a beam being transmitted through the sample.

\section{Conclusions}

In conlusion we would like to point out that the detection scheme has the unique feature of being sensitive to the helicity of radiation. Combining CPGE detection with LPGE one, which is sensitive to the state of linear polarization [16], the ellipticity and the orientation of the ellipse of elliptically polarized radiation can be obtained. Finally we note that the CPGE may also occur at valence-to-conduction band transitions [14] as well at direct intersubband transitions e.g. in the case of asymmetrical (113)-grown $p$-type Si/SiGe structures [17]. Thus the applicability of the CPGE detection scheme may well be extended to the near infrared and mid infrared spectral ranges.

\section{Acknowledgements}

Financial support from the DFG, the RBRF, and the NATO linkage program is gratefully acknowledged. 


\section{References}

[1] A. Hadni, Infrared and Millimeter Waves, vol. 3, eds. K.J. Button and J.C. Wiltse, p. 112 (Academic Press, 1980).

[2] P.M. Valov, K.V. Goncharenko, Yu.V. Markov, V.V. Pershin, S.M. Ryvkin, and I.D. Yaroshetskii, Sov. J. Quantum Electronics 7, 50 (1977).

[3] A.F. Gibson, and M.F. Kimmitt, Infrared and Millimeter Waves, vol. 3, eds. K.J. Button and J.C. Wiltse, p. 182 (Academic Press, 1980).

[4] S.D. Ganichev, Ya.V. Terent'ev, and I.D. Yaroshetskii, Sov. Tech. Phys. Lett. 11, 20 (1985).

[5] M.F. Kimmitt, Infrared Physics 32, 213 (1992).

[6] S.D. Ganichev, S.A. Emel'yanov, A.G. Pakhomov, Ya.V. Terent'ev, and I.D. Yaroshetskii, Sov. Tech. Phys. Lett. 11, 377 (1985).

[7] H. Schneider, C. Schönbein, G. Bihlmann, P. van Son, and H. Sigg, Appl. Phys. Lett. 70, 1602 (1997).

[8] H. Sigg, M.H. Kwakernaak, B. Margotte, D. Erni, P. van Son, and K. Köhler, Appl. Phys. Lett. 67, 2827 (1995).

[9] R.J. Stone, J.G. Michels, S.L. Wong, C.T. Foxton, R.J. Nicholas, and A.M. Fox, Appl. Phys. Lett. 69, 3569 (1996).

[10] S. Winnerl, W. Seifert, E. Schomburg, J. Grenzer, and K.F. Renk, C.J.G.M. Langerak, A.F.G. van der Meer, D.G. Pavel'ev, Yu. Koschurinov, A.A. Ignatov, B. Melzer, V. Ustinov, S. Ivanov, and P.S. Kop'ev, Appl. Phys. Lett. 73, 2983 (1998).

[11] S.D. Ganichev, H. Ketterl, W. Prettl, E.L. Ivchenko, and L.E. Vorobjev, Appl. Phys. Lett. 77, 3146 (2000).

[12] S.D. Ganichev, E.L. Ivchenko, S.N. Danilov, J. Eroms, W. Wegscheider, D. Weiss, and W. Prettl, Phys. Rev. Lett. 86, 4358 (2001).

[13] E.L. Ivchenko and G.E. Pikus, Superlattices and Other Heterostructures. Symmetry and Optical Phenomena, Springer Series in Solid State Sciences, vol. 110, Springer-Verlag, 1995; Ch. 10.

[14] S.D. Ganichev, E. L. Ivchenko, and W. Prettl, Physica E, (in press).

[15] S.D. Ganichev, Physica B 273-274, 737 (1999).

[16] A.V. Andrianov, E.V. Beregulin, S.D. Ganichev, K.Yu. Glukh and I.D. Yaroshetskii, Sov. Tech. Phys. Lett. 14, 580 (1988).

[17] S.D. Ganichev, F.-P. Kalz, U. Rössler, W. Prettl, E.L. Ivchenko, V.V. Bel'kov, R. Neumann, K. Brunner, and G. Abstreiter, Proc. of MRS Fall Meeting, Boston (2001) (in press). 